Pacific Journal of Mathematics

ON GROUPS WITH SPECIFIED LOWER CENTRAL SERIES
QUOTIENTS 


\title{
ON GROUPS WITH SPECIFIED LOWER CENTRAL SERIES QUOTIENTS
}

\author{
JERROLD W. GRossmaN
}

\begin{abstract}
Intrinsic necessary and sufficient conditions are established for a tower of groups to be the tower of lower central series quotients $\left\{G / \Gamma_{s} G\right\}$ of some group $G$, in the case in which $G / \Gamma_{2} G$ is finitely generated and the case in which $G$ is free. A process for constructing a large number of groups with the same lower central series quotient tower is also described.
\end{abstract}

1. Introduction. Given a group $G$, one can form nilpotent approximations $G / \Gamma_{s} G$ to $G$, where $\Gamma_{s} G$ is the normal subgroup of $G$ generated by all simple $s$-fold commutators $(s=1,2, \cdots)$. The tower formed by these lower central series quotients and the natural projections $G / \Gamma_{s+1} G \rightarrow G / \Gamma_{s} G$ deserves the title nilpotent completion tower, or simply completion, of $G$. We do not take the inverse limit of the tower, but rather view the tower either as a diagram or, preferably, as a pro-group. A. K. Bousfield [3] has studied the properties of a transfinite extension of this tower (generalized to incorporate a ring of "coefficients") with application to homological properties of topological spaces. G. Baumslag [2] has investigated groups which have the same completion as a free group. In this paper we study the following problems: Under what conditions is a tower of groups $\left\{G_{s}\right\}$ the completion of some group? Under these conditions, find (all) groups $G$ such that $G_{s} \cong G / \Gamma_{s} G$.

Our principal results are as follows. Call a tower of groups $\left\{G_{s}\right\}$ a $\Gamma$-tower if, for each $s \geqq 1$, the sequence $1 \rightarrow \Gamma_{s} G_{s+1} \rightarrow G_{s+1} \rightarrow G_{s} \rightarrow 1$ is exact. If $\left\{G_{s}\right\}$ is a $\Gamma$-tower and $G_{2}$ is finitely generated, then $\left\{G_{s}\right\}$ is the completion of its inverse limit and, more generally, of each of a transfinite sequence of subgroups of its inverse limit. In particular, we obtain a large number of examples of parafree groups [2]. If $\left\{G_{s}\right\}$ is a $\Gamma$-tower, $G_{2}$ is free abelian, and $\left\{H_{2} G_{s}\right\}$ has trivial projections, then $\left\{G_{s}\right\}$ is the completion of a free group. We do not yet know if every $\Gamma$-tower is the completion of a group.

In $\S 2$ we review pro-groups and establish the basic properties of the completion functor. In $\S 3$ we derive the properties of $\Gamma$-towers. A "decompletion" process in described in $\S 4$, which enables us to construct groups of small cardinality with a given completion, once one group with the given completion is known. We treat the finitely generated case in $\S 5$ and the free case in $\S 6$.

2. Pro-groups and the completion functor. Let $\mathscr{C}$ be any 
category. The category tow- $\mathscr{C}$ has as objects towers in $\mathscr{C}$,

$$
\cdots \longrightarrow X_{s+1} \longrightarrow X_{s} \longrightarrow \cdots \longrightarrow X_{1},
$$

written $\left\{X_{s}\right\}$ and called pro-objects over $\mathscr{C}$ (pro-groups in case $\mathscr{C}$ is the category of groups). The morphisms $X_{s+1} \rightarrow X_{s}$ within the tower (and their compositions) are called projections. Morphisms in tow- $\mathscr{C}$ are given by .

$$
\operatorname{Hom}_{\text {tow }-\mathscr{E}}\left(\left\{X_{s}\right\},\left\{Y_{s}\right\}\right)=\underset{j}{\lim } \underset{i}{\lim } \operatorname{Hom},\left(X_{i}, Y_{j}\right) .
$$

For our purposes it is enough to note that a sequence of morphisms $\left\{X_{s} \rightarrow Y_{s}\right\}$ commuting with the projections in the towers $\left\{X_{s}\right\}$ and $\left\{Y_{s}\right\}$, that is, a morphism in the diagram category, represents a morphism from $\left\{X_{s}\right\}$ to $\left\{Y_{s}\right\}$ in tow- $\mathscr{C}$ and that cofinal towers are isomorphic. See [1], [4], or [7] for a fuller discussion of pro-objects. Although the category of pro-groups is, as we shall see (2.2), the "right" setting in which to study completions, the reader may view the towers in this paper simply as diagrams.

We consider $\mathscr{C}$ as a full subcategory of tow- $\mathscr{C}$ by identifying an object $X$ in $\mathscr{C}$ with the tower $\left\{X_{s}\right\}$ in which each $X_{s}$ is $X$ and each projection the identity. A pro-object isomorphic to an element of $\mathscr{C}$ is called constant. The inclusion functor $\mathscr{C} \rightarrow$ tow- $\mathscr{C}$ is left adjoint to the inverse limit functor $\lim :$ tow $\mathscr{C} \rightarrow \mathscr{C}$, if the latter exists. In that case, $\left\{X_{s}\right\}$ is constant $\overleftarrow{\text { if }}$ and only if $\left\{X_{s}\right\} \cong \lim X_{s}$

We next define the completion functor. Recall [9, Chapter 5] that if $A$ and $B$ are subgroups of a group $G$, then $[A, B]$ denotes the subgroup of $G$ generated by all commutators $[a, b]=a^{-1} b^{-1} a b$ for $a \in A, b \in B$. Inductively define the lower central series of $G$ by $\Gamma_{1} G=G, \Gamma_{s+1} G=\left[\Gamma_{s} G, G\right]$. Thus $\Gamma_{s} G$ is generated by all simple $s$-fold commutators $\left[g_{1}, g_{2}, \cdots, g_{s}\right]=\left[\left[\cdots\left[g_{1}, g_{2}\right], g_{3}\right] \cdots, g_{s}\right]$ of elements of $G$. Let $\Gamma_{\omega} G=\bigcap_{s=1}^{\infty} \Gamma_{s} G$. A group is nilpotent if $\Gamma_{s} G=0$ for some $s<\omega$ and residually nilpotent if $\Gamma_{\omega} G=0$. Each $\Gamma_{s} G$ is normal in $G ; G / \Gamma_{s} G$ is nilpotent for $s<\omega$ and $G / \Gamma_{\omega} G$ is residually nilpotent. The inclusions $\Gamma_{s+1} G \subset \Gamma_{s} G$ give rise to epimorphisms $G / \Gamma_{s+1} G \rightarrow G / \Gamma_{s} G$, and we call the pro-group $\left\{G / \Gamma_{s} G\right\}$ the completion of $G$. Denoting the category of groups [resp. nilpotent groups] by $\mathscr{G}$ [resp. $\mathscr{N}$ ], we more generally define the completion functor $C:$ tow- $\mathscr{G} \rightarrow$ tow- $\mathscr{N}$.

Definition 2.1. Let $\left\{G_{s}\right\} \in$ tow- $\mathscr{G}$. Then $C\left\{G_{s}\right\}$ is the pro-group $\left\{G_{s} / \Gamma_{s} G_{s}\right\}$, called the completion of $\left\{G_{s}\right\}$. There is a canonical morphism $\left\{G_{s}\right\} \rightarrow C\left\{G_{s}\right\}$ induced by the identity.

The proofs of the following propositions are fairly straightforward and hence omitted. 
Proposition 2.2. $\boldsymbol{C}$ is left adjoint to the inclusion functor tow- $\mathscr{N} \rightarrow$ tow- $\mathscr{G}$, and $\boldsymbol{C}$ restricted to $\mathscr{G}$ is left adjoint to the inverse limit functor from tow- $\mathscr{N}$ to $\mathscr{G}$. Furthermore $\left\{G_{s}\right\} \rightarrow C\left\{G_{s}\right\}$ is an isomorphism if and only if $\left\{G_{s}\right\}$ is isomorphic to a tower of nilpotent groups.

Proposition 2.3. For any group $G$,

(i) $1 \rightarrow \Gamma_{s}\left(G / \Gamma_{s+1} G\right) \rightarrow G / \Gamma_{s+1} G \rightarrow G / \Gamma_{s} G \rightarrow 1$ is exact for each $s<\omega$

(ii) $\Gamma_{i}\left(G / \Gamma_{s} G\right) \cong \Gamma_{i} G / \Gamma_{s} G$ for $i \leqq s \leqq \omega ;$

(iii) $\left(G / \Gamma_{s} G\right) / \Gamma_{i}\left(G / \Gamma_{s} G\right) \cong G / \Gamma_{i} G$ for $i \leqq s \leqq \omega$.

3. $\Gamma$-towers. By 2.2 every tower of nilpotent groups is, up to isomorphism in tow- $\mathscr{G}$, its own completion. Our problem is to characterize those towers which are completions of groups.

Definition 3.1. A $\Gamma$-tower is a tower of groups $\left\{G_{s}\right\}$ such that, for each $s \geqq 1$, the sequence

$$
1 \longrightarrow \Gamma_{s} G_{s+1} \longrightarrow G_{s+1} \longrightarrow G_{s} \longrightarrow 1
$$

is exact.

Proposition 3.2. Let $\left\{G_{s}\right\}$ be a $\Gamma$-tower. Then for each s,

(i) $1 \rightarrow \Gamma_{i} G_{\mathrm{s}} \rightarrow G_{\mathrm{s}} \rightarrow G_{i} \rightarrow 1$ is exact for all $i<s$;

(ii) $G_{s} / \Gamma_{i} G_{s} \cong G_{i}$ for all $i<s$;

(iii) $\Gamma_{s} G_{s} \cong 1$;

(iv) if $\Gamma_{s} G_{s+1}=1$, then $G_{k} \cong G_{s}$ for all $k>s$;

(v) if $P$ is a set of generators of $G_{2}$ and $P^{\prime}$ is a set of elements of $G_{s}$ which maps onto $P$ by the projection $G_{s} \rightarrow G_{2}$, then $P^{\prime}$ generates $G_{s}$.

Proof. We prove (i) by induction on $s-i$. The statement is true by definition when $s-i=1$. Denote the projection $G_{m} \rightarrow G_{n}$ by $p_{m, n}$ for $m>n$. Clearly $p_{s, i}$ is surjective; we must show that $\Gamma_{i} G_{s}=\operatorname{ker} p_{s, i}$. Let $x \in \Gamma_{i} G_{s}$. Then $p_{s, s-1}(x) \in \Gamma_{i} G_{s-1}$, so by induction $p_{s, s-1}(x) \in \operatorname{ker} p_{s-1, i}$, whence $x \in \operatorname{ker} p_{s, i}$. Conversely, suppose $x \in \operatorname{ker} p_{s, i}$. Then $p_{s, s-1}(x) \in \operatorname{ker} p_{s-1, i}$. By induction $p_{s, s-1}(x) \in \Gamma_{i} G_{s-1}$; thus we can write $p_{s, s-1}(x)=\prod_{j=1}^{N}\left[a_{j, 1}, a_{j, 2}, \cdots, a_{j, i}\right]$. Since $p_{s, s-1}$ is surjective, we can choose $b_{j, l} \in G_{s}$ such that $p_{s, s-1}\left(b_{j, l}\right)=a_{j, l}$ for $1 \leqq j \leqq N, 1 \leqq l \leqq i$. Let $y=\prod_{j=1}^{N}\left[b_{j, 1}, b_{j, 2}, \cdots, b_{j, i}\right]$. Then $x y^{-1} \in \operatorname{ker} p_{s, s-1}=\Gamma_{s-1} G_{s} \subset \Gamma_{i} G_{s}$. But $y \in \Gamma_{i} G_{s}$, so $x \in \Gamma_{i} G_{s}$. Clearly (i) implies (ii), and (iii) is immediate from the definition. To prove (iv), note that the natural surjection $G_{k} / \Gamma_{s+1} G_{k} \rightarrow G_{k} / \Gamma_{s} G_{k}$ induces an isomorphism $G_{s+1} \rightarrow G_{s}$ by (ii) and the hypothesis; hence $\Gamma_{s+1} G_{k} \cong \Gamma_{s} G_{k}$. But then the definition of the 
lower central series and (iii) imply that $\Gamma_{s} G_{k} \cong \Gamma_{k} G_{k} \cong 1$. Hence by (ii), $G_{s} \cong G_{k} / \Gamma_{s} G_{k} \cong G_{k}$. Finally (v) follows from [9, Lemma 5.9].

By 2.3 (i) $C G$ is a $\Gamma$-tower for every group $G$. We conjecture the converse: Given a $\Gamma$-tower $\left\{G_{s}\right\}$, there exists a group $G$ such that $G / \Gamma_{s} G \cong G_{s}$.

In $\S 5$ we prove this conjecture in case $G_{2}$ is finitely generated, and in $\S 6$ we prove it in case $G_{2}$ is free abelian and $\left\{H_{2} G_{s}\right\} \cong 0$.

4. Constructing small decompletions. If $C G=\left\{G_{s}\right\}$, then the natural map $G \rightarrow \lim G_{s}$ has kernel $\Gamma_{\omega} G$. By 2.3 (iii) the residually nilpotent group $G \overleftarrow{\Gamma_{\omega}} G$ has the same completion as $G$. We therefore make the following definition.

Definition 4.1. Let $\left\{G_{s}\right\}$ be a $\Gamma$-tower. A subgroup $G$ of $\lim G_{s}$ is a proper decompletion of $\left\{G_{s}\right\}$ if the natural maps $G \rightarrow G_{s}$ induce isomorphisms $G / \Gamma_{s} G \cong G_{s}$ for all $s$.

Aside from the case in which a $\Gamma$-tower $\left\{G_{s}\right\}$ is constant (and hence itself its only proper decompletion), $\lim G_{s}$ is uncountable because each surjection $G_{s+1} \rightarrow G_{s}$ has nontrivial kernel by 3.2 (iv). We shall see in the next section that $\lim _{\leftarrow} G_{s}$ is a proper decompletion of $\left\{G_{s}\right\}$ if $G_{2}$ is finitely generated, but $\overleftarrow{w}$ now describe a process for obtaining decompletions with small cardinality.

Proposition 4.2. Let $H$ be a proper decompletion of a nonconstant $\Gamma$-tower $\left\{G_{s}\right\}$. Let $K$ be a subset of $H$. Let $\mathfrak{m}$ be the maximum of the cardinality of $K$, the cardinality of $G_{2}$, and $\boldsymbol{\aleph}_{0}$. Then there exists a proper decompletion of $\left\{G_{s}\right\}$ containing $K$, contained in $H$, and of cardinality $\mathfrak{m}$.

Proof. We shall construct an increasing sequence of subgroups, $A_{1} \subset A_{2} \subset \cdots$, of $H$, each of which is obtained from the preceding one by adjoining at most $m$ elements of $H$, and whose union is the desired decompletion. For each element $g$ in a generating set for $G_{2}$, let $x_{g} \in H$ map to $g$ under the natural surjection $H \rightarrow G_{2}$. Let $A_{1}$ be the subgroup of $H$ generated by $K$ and all the $x_{g}$ 's. Since $A_{1} \rightarrow G_{2}$ is surjective, $A_{1} \rightarrow G_{s}$ is surjective for all $s$ by $3.2(\nabla)$, and the cardinality of $A_{1}$ is $\mathrm{m}$. Assume by induction that we have defined $A_{n} \subset H$ such that $A_{n}$ has cardinality $\mathfrak{m}$ and $A_{n} \rightarrow G_{s}$ is surjective for all $s$. Consider the groups $K_{s}=\operatorname{ker}\left(A_{n} \rightarrow G_{s}\right)$. Clearly $\Gamma_{s} A_{n} \subset K_{s}$, since $\Gamma_{s} G_{s}=1$ by 3.2 (iii), but it might happen that there are elements in $K_{s}$ which are not in $\Gamma_{s} A_{n}$. Such elements are in $\Gamma_{s} H$, however, since $H$ is a proper decompletion of $\left\{G_{s}\right\}$. Form $A_{n+1}$ as 
the subgroup of $H$ generated by $A_{n}$ and a collection of at most $\mathfrak{m}$ elements of $H$ needed to express all the elements of $K_{s}$ as products of simple $s$-fold commutators, for all $s$. Clearly $A_{n+1}$ satisfies the inductive hypotheses. Then $A=\mathrm{U}_{n=1}^{\infty} A_{n}$ is perforce the desired decompletion.

Proposition 4.3. The union of a nested family of proper decompletions of a $\Gamma$-tower is again a proper decompletion.

The proof is clear.

5. The finitely generated case. In this section we use a lemma of Bousfield [3] to show that $\Gamma$-towers with finitely generated $G_{2}$ are actually completion towers, and we construct many decompletions of them. In view of $3.2(\mathrm{v})$, it makes sense to call such a tower a finitely generated $\Gamma$-tower.

THEOREM 5.1. Let $\left\{G_{s}\right\}$ be a finitely generated $\Gamma$-tower, and let $\widehat{G}=\lim _{\leftarrow} G_{s}$. Then $\hat{G}$ is a proper decompletion of $\left\{G_{s}\right\}$.

The proof involves the notion of $N$-series [3], [9, p. 391].

Definition 5.2. An $N$-series in a group $G$ is a descending series of subgroups (indexed by positive integers)

$$
G=K_{1} \supset K_{2} \supset K_{3} \supset \cdots
$$

such that $\left[K_{r}, K_{s}\right] \subset K_{r+s}$ for all $r, s$. There is an associated Lie ring $\bigoplus_{r \geqq 1} K_{r} / K_{r+1}$ with Lie product

$$
\text { [ , ]: } K_{r} / K_{r+1} \otimes K_{s} / K_{s+1} \longrightarrow K_{r+s} / K_{r+s+1}
$$

induced by the commutator.

Lemma 5.3 (Bousfield [3]). Let $\left\{K_{s}\right\}$ be an N-series in a group G such that

(i) the natural $\operatorname{map} G \rightarrow \lim G / K_{s}$ is an isomorphism;

(ii) the Lie product

$$
\text { [ , ]: } G / K_{2} \otimes K_{s} / K_{s+1} \longrightarrow K_{s+1} / K_{s+2}
$$

is surjective for all $s$; and

(iii) $G / K_{2}$ is finitely generated. Then $K_{s}=\Gamma_{s} G$ for all $s \geqq 1$.

Proof of 5.1. Let $K_{s}=\operatorname{ker}\left(\hat{G} \rightarrow G_{s}\right)$. It suffices to show that $\left\{K_{s}\right\}$ is an $N$-series in $\hat{G}$ satisfying the conditions of 5.3. Express 
elements of $\hat{G}$ as sequences $\left(g_{1}, g_{2}, \cdots\right)$ such that $g_{i} \in G_{i}$ and $g_{i+1}$ projects to $g_{i}$ for all $i$. Then $K_{s}=\left\{\left(g_{1}, g_{2}, \cdots\right) \in \widehat{G}: g_{i}=0\right.$ for $\left.i \leqq s\right\}=$ $\left\{\left(g_{1}, g_{2}, \cdots\right) \in \hat{G}: g_{i} \in \Gamma_{s} G_{i}\right.$ for all $\left.i\right\}$ by 3.2 (i) and 3.2 (iii). Since $\left[\Gamma_{r} G_{i}, \Gamma_{s} G_{i}\right] \subset \Gamma_{r+s} G_{i}$ for all $i\left[9\right.$, p. 293], $\left[K_{r}, K_{s}\right] \subset K_{r+s}$. Conditions (i) and (iii) of 5.3 are given. To verify condition (ii), let $\bar{g}=$ $\left(g_{1}, g_{2}, \cdots\right) \in K_{s+1}$. Then $g_{s+2} \in \Gamma_{s+1} G_{s+2}$, so $g_{s+2}=\prod_{j=1}^{N}\left[y_{j, s+2}, z_{j, s+2}\right]$ for some elements $y_{j, s+2} \in \Gamma_{s} G_{s+2}$ and $z_{j, s+9} \in G_{s+2}$. Since $\left\{G_{s}\right\}$ is a tower of surjections, we may extend to $\bar{y}_{j}=\left(y_{j, 1}, y_{j, 2}, \cdots\right) \in K_{s}$ and $\bar{z}_{j}=$ $\left(z_{j, 1}, z_{j, 2}, \cdots\right) \in \hat{G}$. Then $\bar{g}$ and $\Pi_{s=1}^{N}\left[\bar{y}_{j}, \bar{z}_{j}\right]$ differ only by an element of $K_{s+2}$, so the Lie product is onto $K_{s+1} / K_{s+2}$.

Combining 5.1 with 4.2 and 4.3 we can construct inductively a transfinite sequence of decompletions as follows. Let $\left\{G_{s}\right\}$ and $\widehat{G}$ be as in 5.1, with $\left\{G_{s}\right\}$ not constant. Apply 4.2 to the empty subset of $\hat{G}$ to obtain a countable proper decompletion $G^{1}$. Given the proper decompletion $G^{\alpha}$, for an ordinal $\alpha$, if $G^{\alpha} \neq \widehat{G}$, let $x \in \widehat{G}-G^{\alpha}$ and apply 4.2 to $G^{\alpha} \cup\{x\}$ to obtain a proper decompletion $G^{\alpha+1}$, containing, but of the same cardinality as, $G^{\alpha}$. For limit ordinals $\lambda$, let $G^{\lambda}=$ $\mathrm{U}_{\alpha<\lambda} G^{\alpha}$, which is a proper decompletion by 4.3. Note that $G^{\alpha}$ is countable for $\alpha<\omega$ and has cardinality equal to the cardinality of $\alpha$ for $\alpha \geqq \omega$. This process terminates at $\hat{G}$, which has the cardinality of the continuum, $\sqrt{ }$. Although there is no guarantee that the $G^{\alpha}$ 's are not isomorphic, any two with different cardinality will be nonisomorphic, and every cardinality between $\boldsymbol{\aleph}_{0}$ and $\mathfrak{F}$, inclusive, is represented. Since it is consistent to assume [5] that $\mathbb{E}$ is an arbitrarily large cardinal, we have proved the following existence theorem.

THEOREM 5.4. Let $\left\{G_{s}\right\}$ be a nonconstant finitely generated $\Gamma$ tower, and let $\aleph_{\alpha}$ be the $\alpha$ th infinite cardinal number. Then it is consistent with ZFC (set theory plus the axiom of choice) that there exist $\aleph_{\alpha}$ nonisomorphic, residually nilpotent groups with completion $\left\{G_{s}\right\}$.

Letting $\left\{G_{s}\right\}$ be the completion of a finitely generated free group, we obtain a "large number" of examples of parafree groups [2].

6. Completions of free groups. In this section we completely characterize those towers which are completions of (not necessarily finitely generated) free groups. We first need two basic results relating group homology and completion. (These propositions lead Bousfield [3] to call a certain transfinite extension of $\left\{G / \Gamma_{s} G\right\}$ the homological localization tower for $G$.) Given a pro-group $\left\{G_{s}\right\}$ and an integer $n \geqq 1$, define $H_{n}\left\{G_{s}\right\}$ to be the pro-abelian-group $\left\{H_{n} G_{s}\right\}$, where $H_{n} G_{s}$ is the ordinary homology of the group $G_{s}$ with trivial 
integer coefficients $\left[8\right.$, p. 290]. In particular $H_{1}\left\{G_{s}\right\} \cong\left\{G_{s} / \Gamma_{2} G_{s}\right\}$.

Proposition 6.1 (W. G. Dwyer). If $\left\{G_{s}\right\} \rightarrow\left\{G_{s}^{\prime}\right\}$ is a morphism of pro-groups which induces an isomorphism $H_{1}\left\{G_{s}\right\} \rightarrow H_{1}\left\{G_{s}^{\prime}\right\}$ and an epimorphism $H_{2}\left\{G_{s}\right\} \rightarrow H_{2}\left\{G_{s}^{\prime}\right\}$, then $\boldsymbol{C}\left\{G_{s}\right\} \rightarrow C\left\{G_{s}^{\prime}\right\}$ is an isomorphism.

The proof [6] is similar to the proof of the classical version of the theorem due to J. Stallings [10].

Proposition 6.2. Let $\left\{G_{s}\right\}$ be a pro-group. Then the natural morphism $\left\{G_{s}\right\} \rightarrow C\left\{G_{s}\right\}$ induces an isomorphism $H_{1}\left\{G_{s}\right\} \rightarrow H_{1} C\left\{G_{s}\right\}$ and an epimorphism $H_{2}\left\{G_{s}\right\} \rightarrow H_{2} C\left\{G_{s}\right\}$.

Proof. $H_{1} G_{s} \cong H_{1}\left(G_{s} / \Gamma_{s} G_{s}\right)$ by 2.3 (iii). By [10], for each $s$ the short exact sequence

$$
1 \longrightarrow \Gamma_{s} G_{s} \longrightarrow G_{s} \longrightarrow G_{s} / \Gamma_{s} G_{s} \longrightarrow 1
$$

gives rise to a natural exact sequence

$$
H_{2} G_{s} \longrightarrow H_{2}\left(G_{s} / \Gamma_{s} G_{s}\right) \longrightarrow \Gamma_{s} G_{s} / \Gamma_{s+1} G_{s} \text {. }
$$

That $H_{2}\left\{G_{s}\right\} \rightarrow H_{2} C\left\{G_{s}\right\}$ is an epimorphism now follows by forming the corresponding exact sequence of towers and noting that $\left\{\Gamma_{s} G_{s} / \Gamma_{s+1} G_{s}\right\} \cong$ 0 because each projection is the trivial homomorphism.

THEOREM 6.3. Let $\left\{G_{s}\right\}$ be a nonconstant $\Gamma$-tower. Then $\left\{G_{s}\right\}$ has a free group as a proper decompletion if and only if $G_{2}$ is free abelian and $H_{2}\left\{G_{s}\right\} \cong 0$.

Proof. The first condition is clearly necessary, and the second follows from 6.2 since $H_{2} F=0$ for $F$ free. To show sufficiency, let $F$ be the free group on a set of free abelian generators for $G_{2}$, and let $\varphi_{2}: F \rightarrow G_{2}$ be induced by the identity. Lift $\varphi_{2}$ to a morphism $\varphi: F \rightarrow\left\{G_{s}\right\}$. By 3.2 (ii) and the hypothesis that $H_{2}\left\{G_{s}\right\} \cong 0, H_{1} \varphi$ is an isomorphism and $H_{2} \varphi$ is an epimorphism. Hence $\boldsymbol{C} \varphi$ is an isomorphism by 6.1. In fact a diagram chase, using the characterization of isomorphism in tow- $\mathscr{G}$ in [4], shows that each level $F / \Gamma_{s} F \rightarrow G_{s}$ of $\boldsymbol{C} \phi$ is an isomorphism. Finally since free groups are residually nilpotent [2], the image of $F$ in $\lim G_{s}$ is free and a proper decompletion of $\left\{G_{s}\right\}$. 


\section{REFERENCES}

1. M. Artin and B. Mazur, Etale Homotopy, Lecture Notes in Mathematics, No. 100, Springer-Verlag, Berlin-New York, 1969.

2. G. Baumslag, Groups with the same lower central sequence as a relatively free group. I. The groups, Trans. Amer. Math. Soc., 129 (1967), 308-321.

3. A. K. Bousfield, Homological localization towers for groups and $\pi$-modules, Memoirs of the Àmer. Math. Soc., 10 (1977), No. 186.

4. A. K. Bousfield and D. M. Kan, Homotopy Limits, Completions and Localizations, Lecture Notes in Mathematics, No. 304, Springer-Verlag, Berlin-New York, 1972.

5. P. J. Cohen, Set Theory and the Continuum Hypothesis, Benjamin, New York, 1966.

6. W. G. Dwyer, Homology decomposition towers, preprint, 1972.

7. J. W. Grossman, Homotopy groups of pro-spaces, Illinois J. Math., 20 (1976), 622-625.

8. S. MacLane, Homology, Springer-Verlag, Berlin-New York, 1963.

9. W. Magnus, A. Karrass, and D. Solitar, Combinatorial Group Theory, Interscience, New York, 1966.

10. J. Stallings, Homology and central series of groups, J. Algebra, 2 (1965), 170-181.

Received November 29, 1976 and in revised form August 4, 1977.

OAKLAND UNIVERSITY

ROCHESTER, MI 48063 


\section{PACIFIC JOURNAL OF MATHEMATICS}

\section{EDITORS}

RICHARD ARENS (Managing Editor)

University of California

Los Angeles, California 90024

C. W. Curtis

University of Oregon

Eugene, OR 97403

C. C. MOORE

University of California

Berkeley, CA 94720

\section{J. DUGUNDJI}

Department of Mathematics University of Southern California Los Angeles, California 90007

R. Finn AND J. Milgram Stanford University Stanford, California 94305

ASSOCIATE EDITORS
E. F. BECKENBACH

B. H. NeUMANN

F. WOLF

K. YoshidA

\section{SUPPORTING INSTITUTIONS}

UNIVERSITY OF BRITISH COLUMBIA UNIVERSITY OF SOUTHERN CALIFORNIA CALIFORNIA INSTITUTE OF TECHNOLOGY STANFORD UNIVERSITY UNIVERSITY OF CALIFORNIA MONTANA STATE UNIVERSITY UNIVERSITY OF TOKYO UNIVERSITY OF NEVADA, RENO UNIVERSITY OF UTAH NEW MEXICO STATE UNIVERSITY OREGON STATE UNIVERSITY UNIVERSITY OF OREGON

WASHINGTON STATE UNIVERSITY UNIVERSITY OF WASHINGTON OSAKA UNIVERSITY 


\section{Pacific Journal of Mathematics}

\section{Vol. 74, No. $1 \quad$ May, 1978}

Gerald Arthur Anderson, Computation of the surgery obstruction groups

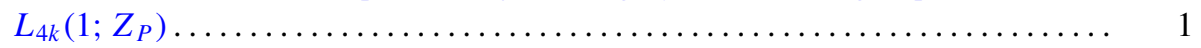

R. K. Beatson, The degree of monotone approximation ................ 5

Sterling K. Berberian, The character space of the algebra of regulated functions . . . 15

Douglas Michael Campbell and Jack Wayne Lamoreaux, Continua in the plane with

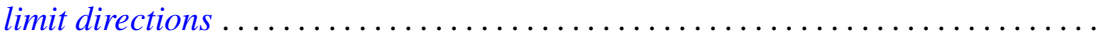

R. J. Duffin, Algorithms for localizing roots of a polynomial and the Pisot

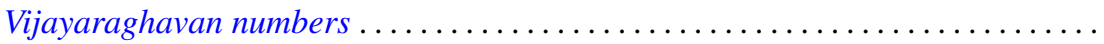

Alessandro Figà-Talamanca and Massimo A. Picardello, Functions that operate on

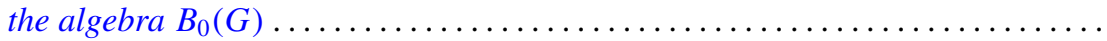

John Erik Fornaess, Biholomorphic mappings between weakly pseudoconvex

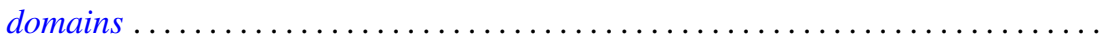

Andrzej Granas, Ronald Bernard Guenther and John Walter Lee, On a theorem of S.

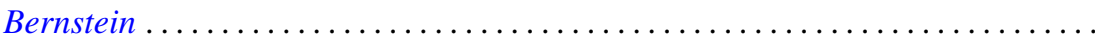

Jerry Grossman, On groups with specified lower central series quotients . .........

William H. Julian, Ray Mines, III and Fred Richman, Algebraic numbers, a constructive development . . . . . . . . . . . . . . . . . . . . . . .

Surjit Singh Khurana, A note on Radon-Nikodým theorem for finitely additive

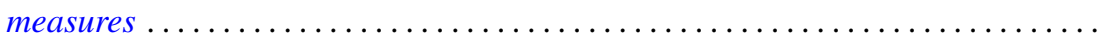

Garo K. Kiremidjian, A Nash-Moser-type implicit function theorem and nonlinear

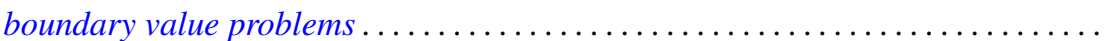

Ronald Jacob Leach, Coefficient estimates for certain multivalent functions ....

John Alan MacBain, Local and global bifurcation from normal eigenvalues. II . . 133

James A. MacDougall and Lowell G. Sweet, Three dimensional homogeneous algebras...

John Rowlay Martin, Fixed point sets of Peano continua ......

R. Daniel Mauldin, The boundedness of the Cantor-Bendixson order of some analytic sets...

Richard C. Metzler, Uniqueness of extensions of positive linear functions ..

Rodney V. Nillsen, Moment sequences obtained from restricted powers . .

Keiji Nishioka, Transcendental constants over the coefficient fields in differential elliptic function fields...

Gabriel Michael Miller Obi, An algebraic closed graph theorem

Richard Cranston Randell, Quotients of complete intersections by $\mathbf{C}^{*}$ actions . . 221

Bruce Reznick, Banach spaces which satisfy linear identities . .

Bennett Setzer, Elliptic curves over complex quadratic fields...

Arne Stray, A scheme for approximating bounded analytic functions on certain subsets of the unit disc.

Nicholas Th. Varopoulos, A remark on functions of bounded mean oscillation and bounded harmonic functions. Addendum to: "BMO functions and the

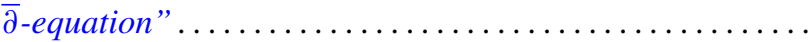

Charles Irvin Vinsonhaler, Torsion free abelian groups quasi-projective over their

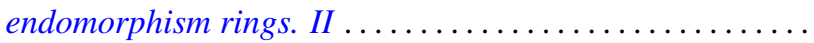

Thomas R. Wolf, Characters of $p^{\prime}$-degree in solvable groups ... 